\title{
e-Phaïstos
}

e-Phaïstos

Revue d'histoire des techniques / Journal of the history

of technology

IV-1 | 2015

Les arts de guerre et de grâce (XIVe-XVIIle siècles)

\section{Pratiquer les AMHE aujourd'hui : entre reconstitution, expérimentation et innovation}

Practicing Historical European Martial Arts today: between reconstitution, testing and innovation

\section{Audrey Tuaillon Demésy}

\section{(2) OpenEdition}

Journals

Édition électronique

URL : http://journals.openedition.org/ephaistos/669

DOI : 10.4000/ephaistos.669

ISSN : 2552-0741

Éditeur

IHMC - Institut d'histoire moderne et contemporaine (UMR 8066)

Édition imprimée

Date de publication : 1 avril 2015

ISSN : 2262-7340

Référence électronique

Audrey Tuaillon Demésy, « Pratiquer les AMHE aujourd'hui : entre reconstitution, expérimentation et innovation », e-Phaïstos [En ligne], IV-1 | 2015, mis en ligne le 01 avril 2015, consulté le 19 avril 2019. URL : http://journals.openedition.org/ephaistos/669; DOI : 10.4000/ephaistos.669

Ce document a été généré automatiquement le 19 avril 2019

Tous droits réservés 


\section{Pratiquer les AMHE aujourd'hui : entre reconstitution, expérimentation et innovation}

Practicing Historical European Martial Arts today: between reconstitution, testing and innovation

Audrey Tuaillon Demésy

1 Les Arts martiaux historiques européens, couramment nommés AMHE par ceux qui les pratiquent à l'heure actuelle, sont une forme d'activité physique et culturelle qui se donne pour objectif de retranscrire des gestes et des techniques propres à un affrontement normé entre un individu et un petit nombre d'adversaires (en général, un seul). Les AMHE se présentent sous la forme d'une recréation de gestes techniques, martiaux, issus de l'espace historique européen. La recherche menée concerne plus spécifiquement les AMHE ayant trait à la période médiévale et au XvI ${ }^{\mathrm{e}}$ siècle, mais il faut souligner que ces pratiques ne sont pas limitées à ces seules époques.

De manière globale, les AMHE prennent place au sein de l'histoire vivante. Une approche socio-ethnographique (mêlant observations participantes, entretiens et questionnaires ethnographiques) a permis de dresser des parallèles entre la reconstitution historique et les $\mathrm{AMHE}^{1}$, qui apparaissent comme deux faces d'une même pratique. En effet, l'histoire vivante est une activité culturelle qui vise à mettre en vie un événement ou des manières de faire d'un temps passé. La re-création, effectuée d'après des recherches et études de sources primaires (pièces de fouilles, manuscrits, etc.), est au fondement de la démarche ainsi réalisée. Pour ce qui est de la reconstitution, il s'agit plus spécifiquement de représenter une personne (ayant ou non existé), comme reflet d'une époque. Ainsi, l'histoire vivante se décline selon deux axes (la reconstitution et les AMHE) qui se rejoignent quant aux thématiques abordées (le Moyen-Âge, la mise en vie d'une action) et aux manières de faire (travail sur les artefacts historiques, re-création, etc.). Plus précisément, les AMHE se comprennent comme une pratique martiale : ce vocabulaire fait directement référence au dieu de la guerre Mars et aux «Arts», compris au sens de 
«science». Le cadre temporel et géographique est par ailleurs clairement identifié (Europe, arts du passé).

3 Au-delà du cadre global dans lequel doivent être appréhendés les AMHE, il s'agit de mettre en avant différentes « façons de faire » des arts martiaux de ce type aujourd'hui ${ }^{2}$. Il existe en effet plusieurs conceptions de l'activité, qui n'englobent pas les mêmes enjeux. Les AMHE seront en premier lieu présentés comme une pratique reconstituée ; puis ils seront abordés sous l'angle d'une démarche expérimentale et enfin, la question de l'innovation sera soulevée.

\section{Les AMHE : une pratique de reconstitution}

Dans un premier temps, il s'agit de donner à voir les AMHE sous l'angle d'une activité mettant en scène les principes d'une reconstitution. Plusieurs éléments permettent de mener l'analyse selon cette orientation. Les AMHE se positionnent au carrefour d'une activité à la fois physique (qui va demander une dépense énergétique importante et recourir à des situations motrices) et culturelle (qui va mobiliser un lien avec l'Histoire). Pour cette raison, elle est d'emblée multiple et plurielle: l'aspect physique va systématiquement être articulé avec le côté culturel.

5 Plus précisément, des recherches sont menées pour comprendre les arts du combat, en lien avec les sources primaires. Ces études peuvent être réalisées par des amateurs (en règle générale, les présidents des clubs d'AMHE, qui sont aussi la plupart du temps les instructeurs principaux de leur groupe), comme par des personnes dont la recherche est l'occupation principale. En ce qui concerne les chercheurs "amateurs", l'enjeu est de pouvoir réutiliser les sources primaires (les données historiques ou archéologiques) pour appliquer, de manière physique, ce qui est exposé dans ces sources. D'autre part, plusieurs pratiquants mènent des recherches dans un cadre davantage professionnalisant. Un lien avec des disciplines universitaires est alors mis en place. En effet, certains présidents d'association ou instructeurs sont aussi doctorants ou jeunes docteurs en histoire ou en archéologie. Le doctorat, réalisé la plupart du temps dans une optique professionnelle, est souvent complété par une pratique physique des AMHE qui est détachée de la recherche. En effet, les travaux menés le sont dans un but scientifique, faisant référence à des critères très précis et à un protocole de recherche, tandis que la pratique est abordée sous l'angle d'une démarche essentiellement physique.

6 Le lien entre ces deux facettes des AMHE, l'aspect recherche et la place laissée aux applications techniques, se comprend vis-à-vis du rapport au corps et des gestes effectués. La question de l'efficience biomécanique est systématiquement placée au centre des recherches. Pour autant, des dissensions peuvent apparaître entre les différents acteurs: le corps peut être considéré comme un outil permettant de reconstituer les techniques martiales historiques ou, au contraire, l'aspect corporel n'est absolument pas envisagé dans le cadre de la pratique contemporaine. Tout se passe alors comme si les recherches menées sur les techniques se dissociaient entre une pratique de loisir, qui fait nécessairement appel au corps, et une pratique fondamentale, qui n'utilise le corps que comme un outil d'expérimentation, sans laisser de place à l'activité physique en tant que telle. La reconstitution des gestes techniques doit se comprendre en lien avec les recherches effectuées en amont. 
7 Toutefois, l'activité physique est nécessairement rattachée à l'activité culturelle : les pratiquants revendiquent cette dualité dans leur activité. L'aspect culturel est essentiellement mobilisé à travers les recherches effectuées et le rapport privilégié entretenu à la «source». Ce terme, fréquemment employé par les pratiquants, fait référence au corpus de documentation disponible et utilisé (souvent, ce sont des traductions et/ou des transcriptions mises à disposition par les «chercheurs » sur les espaces d'échanges numériques), pour mener à bien les recherches ayant trait aux techniques étudiées. Il s'agit d'un support qui va permettre de garantir l'historicité des activités effectuées. Ces «sources » sont donc à appréhender comme des éléments " garants » de la pratique en elle-même en ce que le rapport à l'histoire est respecté. Ce sont bien ces données primaires qui vont autoriser la reconstitution de gestes techniques précis. Cependant, tous les initiés (ce terme doit être compris, ici, au sens de pratiquants réguliers, qui ont connaissance de la «démarche» des AMHE, des sources à l'expérimentation) ne travaillent pas sur ces sources primaires et des traductions, des données de seconde main (souvent réalisées par les chercheurs professionnels) sont utilisées par les instructeurs ou les pratiquants. Elles viennent se substituer à la source primaire, mais permettent malgré tout de garantir une forme de savoir historique, validé par des chercheurs sinon professionnels, du moins confirmés.

8 À ce moment précis de la démarche, c'est la fidélité de la technique par rapport à la source historique (texte ou image), qui apparait comme essentielle. La question de la continuité martiale et corporelle est au centre de la technique mise en œuvre. Les répétitions de gestes sont une part essentielle des savoir-faire spécifiques aux AMHE. L'objectif n'est pas de créer une nouvelle technique, mais de valider des hypothèses théoriques élaborées en amont ou de reproduire un coup. Les AMHE ont alors une double fonction : ils servent de support physique à une analyse scientifique ou permettent la reconstitution d'une pièce ou d'un jeu martial, qui doivent être fidèles à la source prise pour modèle. La recréation des gestes s'apparente à un savoir-faire pratique, qui consiste à présenter une image de la source, remise en vie par le biais des corps des pratiquants. La finalité de la démarche est de représenter le plus fidèlement possible la technique. La plupart du temps, seule une partie de la gestuelle complète est illustrée dans les traités. Il s'agit alors de mener une analyse technique afin de parvenir au résultat escompté, présenté dans les sources.

9 Les AMHE comprennent donc un axe qui vise à reconstituer, au sens de « reformer de nouveau", des gestes comme des techniques martiales historiques. Le but est de présenter, à travers la permanence de la biomécanique corporelle, la faisabilité de certaines " pièces ", coups, etc. Il s'agit de donner à voir des pratiques qui soient fidèles à celles exposées au sein des sources primaires. Pour autant, la simple reconstitution ne suffit pas pour mener à bien des techniques dans leur ensemble, puisque toutes les étapes ne sont pas systématiquement développées dans les sources. Dès lors, une part d'expérimentation semble nécessaire.

\section{L'expérimentation}

10 En dehors de la recréation fidèle aux sources de techniques martiales, l'expérimentation apparait comme un moyen permettant de saisir les différentes étapes d'une succession de mouvements. Les pratiques sont alors réactualisées, replacées dans un contexte contemporain, autorisant les «essais » qui vont servir à finaliser la totalité d'un coup 
porté, l'utilisation des seules sources ne permettant pas de recréer dans leur totalité des techniques entières. En effet, ne sont parfois représentées ou décrites que les situations de fin d'assaut. Dès lors, il convient d'expérimenter avant de parvenir à cette dernière phase.

\section{Les enjeux de l'expérimentation}

11 Les AMHE visent à re-présenter des pratiques martiales : en ce sens, il faut passer de données textuelles ou iconographiques, à la translation de celles-ci à travers une corporéité contemporaine. La source historique est dépassée, les applications vont audelà de sa simple lecture afin de donner vie à la pratique actuelle des arts martiaux européens. L'expérimentation va servir à compléter les éléments manquants dans les documents historiques. Elle est donc une phase nécessaire pour la démarche contemporaine, permettant de lier les "pièces" entre elles et de rendre fluides les enchaînements techniques effectués. La reconstitution est efficiente pour la recherche fondamentale portant sur les arts de combat; par la suite, l'expérimentation s'avère pertinente pour que les pratiquants puissent exécuter les mouvements et se projeter audelà de la simple approche historique figée dans un texte, un artefact ou une iconographie. Des éléments nouveaux par rapport à l'Histoire sont introduits, les gestes ne sont plus uniquement reconstitués mais conduisent à une forme d'expérimentation nouvelle, nécessairement reliée au corps du pratiquant. Pour que la démarche sorte du cadre «culturel» et entre dans le champ d'une activité physique, ce phénomène d'expérimentation est primordial, puisqu'il va permettre la mise en action de gestes et mouvements en dehors du cadre normé et codifié de la source primaire.

Cette étape supplémentaire dans la façon de concevoir les AMHE invite à s'interroger sur la notion "d'authenticité », en particulier historique. Celle-ci révèle ce qui est «vrai » (autrement dit, dans ce cas précis: historiquement avéré, contrôlé par les données primaires). Dès lors, les AMHE, se fondant sur le rapport à la source et à l'historicité des techniques, peuvent-ils encore être considérés comme une activité culturelle, si des écarts par rapport aux données primaires sont constatés et mis en avant?

\section{La recherche d'authenticité}

13 L'écart entre la recherche fondamentale et la pratique physique se comprend à l'aune du regard porté par les initiés quant à l'authenticité de leurs pratiques. Si la reconstitution d'une technique permet la validation d'hypothèses scientifiques, l'expérimentation favorise la réalisation possible de ces arts martiaux, à travers la mise en jeu de techniques corporelle re-créées. L'authenticité se retrouve à plusieurs niveaux, en fonction des attentes de chaque pratiquant. Ainsi, le chercheur se servira des AMHE comme d'un outil, et les initiés, à travers la mise en jeu de la biomécanique humaine, vont rechercher l'authenticité dans une réalité reconstruite, mise en vie. Ce qui importe n'est plus seulement la pièce, le moment présenté dans la source, mais l'ensemble des mouvements permettant de parvenir à un résultat. Il existe une envie de la part des pratiquants de mettre en action une «vraie» technique, c'est-à-dire un ensemble de gestes historiquement avérés et liés à une forme d'ancestralité. La « source » en tant que telle ne pouvant apporter cette garantie historique, d'autres éléments vont permettre de justifier la démarche, tout en autorisant l'inscription des AMHE dans un domaine culturel. 
14 En premier lieu, le processus corporel permettant de reproduire une succession de coups est perçu comme un indicateur efficient de la faisabilité d'une technique. Autrement dit, c'est le rapport au corps, à la biomécanique, qui va permettre de tester la technique et de l'inscrire dans le domaine du possible et, par extension, de l'authentique. Le postulat de départ est que la mécanique corporelle n'a pas changé et qu'un « coude casse toujours de la même façon $»^{3}$, à l'heure actuelle ou au Moyen-Âge. La permanence du corps humain est un facteur supplémentaire, au-delà de la source, qui va favoriser la mise en vie de techniques et leur possible inscription dans un cadre historique. Le passage se fait alors de la reconstitution de gestes à une expérimentation martiale. Les AMHE sont replacés dans un contexte plus global de duels et d'affrontements entre deux adversaires.

La démarche historique des actions mises en œuvre est également garantie par la figure des «instructeurs", qui sont les personnes enseignant et transmettant leurs connaissances aux autres pratiquants. Les instructeurs détiennent en effet une somme de compétences associées à l'historicité des techniques transmises et échangées : ce sont leurs savoirs en matière d'appréhension et d'analyse des sources qui sont au cœur de l'identité qu'ils présentent aux autres membres. Bien que tous ne soient pas chercheurs professionnels, ils ont su développer des «qualités» qui leur assurent un statut particulier au sein du groupe. L'instructeur est en effet celui qui « enseigne » et la transmission se déroule de manière verticale. Ce fait s'observe en particulier au cours des stages: une personne est responsable d'un atelier et expose les techniques à partir desquelles il a travaillé. Pour autant, cette transmission verticale peut aussi se muer en échange, puisque les instructeurs peuvent à leur tour devenir participant au cours d'un même stage. D'enseignants, ils deviennent à leur tour " apprenants ». Les compétences et connaissances de chaque instructeur se développant dans un domaine précis (à propos d'un traité en particulier, d'un auteur, d'une arme, etc.), les échanges sont effectifs parce que les AMHE sont eux-mêmes une pratique plurielle. L'instructeur apparaît comme un garant des techniques enseignées et, par extension, de leur conformité à une source historique. Ceci se comprend d'autant plus que la posture occupée fait souvent référence à une hiérarchie, communément admise dans le système global d'enseignement ou d'éducation. À ce propos, Howard Becker rappelle que «dans sa définition conventionnelle, l'éducation correspond à des gens savants qui enseignent à des gens moins savants, qui, mais cela n'est pas surprenant, sont typiquement moins puissants et moins bien placés $»^{4}$. Les pratiquants eux-mêmes acceptent et revendiquent le statut particulier de leur instructeur : il est le "chef », souvent aussi le "président », etc. En outre, les instructeurs sont souvent les membres référents de leur association. Ils occupent un statut particulier, socialement dominant par rapport aux "simples » pratiquants. La forme prise par la transmission peut se rapprocher, dans une certaine mesure, de celle de l'éducation.

16 L'expérimentation dans les AMHE prend donc une forme double : celle d'une transmission verticale mais aussi celle de l'échange, lorsque les instructeurs se forment auprès d'autres pratiquants. Il est à noter que de plus en plus, avec l'augmentation du nombre d'adhérents, les instructeurs se multiplient au sein des associations, chacun pouvant avoir un thème de recherche particulier. Ce phénomène a tendance à faire disparaître la place centrale occupée par un enseignant unique au sein d'un groupe. 


\section{Une pratique en cours de patrimonialisation?}

17 Un dernier aspect qui touche à l'expérimentation dans les AMHE et qui permet d'inscrire la pratique comme culturelle malgré les écarts à la source, est l'aspect patrimonial inhérent à la démarche.

La notion de patrimoine est polysémique et mérite quelques précisions. Le principe qui sera mobilisé, en lien avec l'histoire vivante en général et avec les AMHE en particulier, est celui d'un héritage commun soumis à conservation. Plus précisément, le patrimoine « désigne l'ensemble des biens communs à une unité de civilisation - région, nation ou même humanité - qui, recensés dans le passé, permettent de fonder, de définir et de réactiver la sensibilité à l'identité collective [...]. La présence d'une menace pesant sur le futur vivifie l'esprit patrimonial $»^{5}$. L'idée de patrimoine fait directement référence aux savoirs historiques qui, mis en œuvre, vont permettre une identification commune du groupe des pratiquants à un passé identique pour tous: l'objectif revendiqué est de présenter une vision différente de celle habituellement associée au combat médiéval. Ainsi, un assaut ne dure que quelques secondes et se place loin des représentations véhiculées par le cinéma.

Ce qui est mis en avant est la « conservation » de données textuelles ou manuelles, mais aussi une forme de remise en vie. Le patrimoine se caractérise par un lien fort avec le passé : les AMHE le mobilisent en lien direct avec la re-création, qui va autoriser l'action motrice et corporelle et permettre de se rapprocher des arts du combat tels qu'ils pouvaient être pratiqués à l'époque. Ainsi, la mise en valeur de gestes techniques au-delà de la simple reproduction à l'identique et de la seule mise en vie d'une image, mais à travers une action motrice, va autoriser l'exposition d'un patrimoine souvent ignoré. Il s'agit de présenter de nouveaux objets patrimoniaux à protéger et sauvegarder. Il est possible, à cet égard, de se référer au patrimoine culturel immatériel, tel qu'il a pu être défini par l'UNESCO ${ }^{6}$. Les savoirs ainsi développés sont directement reliés au patrimoine (" historique européen ») et s'étendent ensuite aux aspects techniques, qui ne peuvent être abordés qu'à travers une action gestuelle (autrement dit, plutôt décomposée) et corporelle (mise en jeu de l'ensemble de la corporéité du pratiquant). Cette facette de l'expérimentation se retrouve lorsque les associations d'AMHE mettent en place des représentations publiques. Les conservateurs de musées, par exemple, peuvent solliciter une association afin que ses membres effectuent des démonstrations (le plus souvent à l'épée, mais pas uniquement). L'enjeu est de passer d'un objet inerte exposé dans une vitrine (une épée, un manuscrit, etc.) à une présentation vivante, forme de continuité des collections muséales. Le lien entre l'objet et le geste est étroit et c'est bien en partant du premier que des applications par expérimentation peuvent être réalisées. Il est alors possible de parler de patrimoine technique : ce sont les façons de faire et les gestes qui sont objets de conservation et de sauvegarde. L'approche culturelle des arts martiaux historiques est garantie par le phénomène de patrimonialisation, bien que l'expérimentation aille au-delà de la simple analyse de la source. Autrement dit, les interprétations contemporaines des manuscrits, suivies de leur application concrète, permettent de renouveler le travail du geste et de retrouver des méthodes de combat perdues quant à leur réalisation matérielle (il peut dans ce cas s'agir de re-création d'armes par le biais des simulateurs), et corporelle. 
20 Ainsi, l'expérimentation est une étape supplémentaire dans la pratique des AMHE, en ce qu'elle mobilise des actions corporelles permettant la mise en action des techniques martiales historiques. Dépassant l'analyse de la source, la question de l'authenticité de la démarche est questionnée. La permanence de l'inscription des AMHE comme activité culturelle se retrouve à différents niveaux. D'abord, dans l'optique d'une permanence bio-mécanique et corporelle qui va autoriser la recréation de gestes; ensuite, à travers les connaissances transmises par les instructeurs qui apparaissent comme les «garants » de la conformité de la pratique par rapport à la source; et enfin, le processus de patrimonialisation des gestes techniques rappelle le fort lien entretenu avec l'envie de conserver des savoir-faire ayant trait aux arts du combat. L'aspect culturel reste au centre de l'expérimentation dans les AMHE, même si la mise en action se positionne du côté de l'activité physique. Cette dernière va cependant se développer davantage sous l'angle de l'innovation dans la démarche.

\section{L'innovation}

21 La reconstitution et l'expérimentation forment les deux premières approches mises en place dans la pratique des AMHE. Pour autant, ces arts du combat peuvent aussi être abordés sous l'angle de l'innovation. Celle-ci se décline selon deux grands axes. D'une part, les AMHE sont effectués comme une pratique moderne, entièrement inscrite dans le monde contemporain. D'autre part, le phénomène de sportivisation doit être pris en compte pour comprendre l'importance prise par la forme physique de la démarche.

\section{Une pratique moderne}

Les AMHE ne sont réellement pratiqués en France que depuis une dizaine d'années, sous l'impulsion des associations loi 1901. Celles-ci forment en effet l'essentiel, pour ne pas dire la totalité, du cadre légal regroupant les activités d'AMHE. De plus, la pratique est comprise comme un moment de détente et de loisir, comme en témoigne l'importance accordée à la bonne ambiance et à la convivialité lors de sessions d'entraînement ou au cours des stages. Le loisir est la forme adoptée de façon spontanée par les pratiquants. Les AMHE s'inscrivent ainsi pleinement dans le cadre d'une société du loisir, mobilisé au sein du temps libre ${ }^{7}$. De façon plus spécifique, «il y a des loisirs passifs qui n'exigent pas d'effort particulier des consommateurs, et puis des loisirs actifs qui, s'ils apportent du plaisir aux individus, demandent un investissement important et améliorent aussi le niveau de capital humain ${ }^{8}$. Cette citation présente un double intérêt : elle rappelle que les loisirs impliquent la consommation et que, par extension, les pratiquants sont aussi des consommateurs. De plus, ces derniers sont inscrits au sein d'un loisir actif, en ce que celui-ci demande une forte participation (présence aux sessions d'entraînement, etc.). Cette forme prise par la pratique induit par la suite le développement d'activités professionnelles. En effet, si les pratiquants sont des consommateurs, ils vont chercher à entrer dans un cycle marchand, qui se caractérise entre autres par l'achat de matériel; d'où l'apparition de vendeurs spécialisés. Le fait de prendre part à un loisir actif permet une évolution constante de celui-ci malgré le nombre relativement faible de participants.

De même, les échanges culturels ayant trait à l'aspect historique des AMHE passent principalement par les outils mis à disposition via l'internet. Les forums des associations et les réseaux sociaux sont des supports d'échanges privilégiés, parce qu'ils permettent 
d'obtenir des réponses rapides à des interrogations exposées, mais également parce que les distances géographiques sont ainsi abolies. De même, les supports vidéo sont fréquemment utilisés. Ils servent souvent à présenter les résultats d'une recherche. Ces images sont présentées sur les forums et les auteurs en attendent souvent des critiques ou des réactions (sur la forme comme sur le fond) de la part des autres pratiquants. Une forme d'approbation est recherchée par le biais des travaux (vidéo, traduction, etc.) partagés. Autrement dit, un phénomène d'auto-évaluation a lieu au sein du groupe des initiés aux AMHE.

Un autre objectif de l'utilisation des espaces numériques est la médiatisation qui peut être faite, par ce biais, des activités. L'internet sert de support à une diffusion de ce que proposent les AMHE. La plupart des associations ont un site internet ou une page facebook, qui agissent comme une vitrine de leur démarche. Une page web ${ }^{9}$ a été consacrée au référencement de tous les groupes français (ou frontaliers). Cet annuaire donne une image globale de la pratique et à oriente ceux qui cherchent à rejoindre cette activité.

Enfin, un autre trait qui inscrit les AMHE comme une activité moderne est le matériel utilisé pour pratiquer. Il s'agit en effet de simulateurs adaptés à une pratique sécurisée. Il faut se protéger soi-même des coups reçus, mais également ne pas blesser son partenaire au cours des exercices. Ainsi, des protections sont adoptées, souvent issues d'autres pratiques physiques ou sportives : masques d'escrime, vestes de maitre d'armes, gants de moto ou de hockey, etc. De même, les outils offensifs sont conçus pour une pratique moderne qui cherche à minimiser la violence : dagues en bois, dussacks en cuir (arme du $\mathrm{XVI}^{\mathrm{e}}$ siècle à une main, possédant un tranchant recourbé), etc. Il est à noter que le matériel se spécialise de plus en plus et s'adapte aux besoins des pratiquants : les épées en nylon remplacent les épées en bois, les équipements défensifs deviennent plus légers et sont spécialement adaptés pour les AMHE, etc. La spécificité de l'activité voit de plus en plus ses besoins, en termes d'outils, comblés par l'apparition de nouveaux marchés. En ce sens, les AMHE innovent par rapport aux sources : la pratique moderne doit répondre à des impératifs de sécurité qui nécessitent des adaptations ${ }^{10}$.

\section{La sportivisation}

Un autre aspect à aborder est le phénomène de sportivisation ${ }^{11}$ qui tend à se mettre en place. Les AMHE sont bien définis comme une activité physique et culturelle mais la question se pose de savoir si cette démarche physique ne tend pas à devenir une activité sportive.

Tout d'abord, il faut mentionner le fait que les AMHE disposent depuis récemment (début 2012) d'une fédération : la Fédération française des AMHE (FFAMHE). Les objectifs de cet organisme sont de mettre en avant la recherche, l'étude et la pratique des AMHE, et d'en faire la promotion. Les AMHE sont présentés par la fédération sous l'angle des « situations motrices employées au combat $»^{12}$. L'aspect sportif n'est pas mentionné et de manière générale, l'un des soucis constants des pratiquants est de distinguer leur activité du sport. De même, la notion de « compétition » est très peu énoncée.

28 Ceci invite à s'interroger sur la définition du sport, qui englobe aujourd'hui presque systématiquement les notions de compétition, de classement ou encore de dépassement de soi. Il est possible de délimiter le sport selon « deux niveaux de définition, un sens restreint où le sport désigne l'ensemble des pratiques physiques, codifiées, institutionnalisées, réalisées en vue d'une performance ou d'une compétition et 
organisées pour garantir l'égalité des conditions de réalisation, et dans un sens plus étendu où il englobe tout type d'activité physique réalisé dans un but récréatif, hygiénique ou compétitif et dans un cadre réglementaire minimal $\aleph^{13}$. Ces définitions du sport impliquent le principe compétitif, qui renvoie à une concurrence, organisée lors de championnats par exemple. D'autres auteurs insistent davantage sur le côté « loisir » de l'activité sportive mais la notion qui importe ici, et celle qui fait le plus souvent débat, est celle de "compétition». C'est donc le sport comme système réglé, codifié, institutionnalisé et faisant appel à une forme de classement, qui sera pris en compte en lien avec les AMHE effectués sous l'angle de l'innovation. Il convient de montrer le glissement qui peut s'opérer de l'activité physique à la démarche sportive.

En dehors de l'expérimentation, certaines techniques (notamment les actions motrices) ne peuvent être enseignées et le pratiquant doit innover, trouver des solutions par luimême pour parvenir à réaliser un geste particulier. Il s'agit, comme le note un instructeur, d'« utiliser son imagination $»^{14}$. Pour ce faire, la méthode de travail à vitesse « réelle » est mise en place : ce sont les sparring. Cette technique consiste à s'équiper de manière à être protégé du mieux possible et à appliquer les gestes appris au préalable. Ces assauts libres viennent compléter les premières phases et apportent au pratiquant des conditions d'affrontements qui ne sont plus celles d'un apprentissage. L'enjeu des assauts est de tester les acquis des participants et de mobiliser une adhésion volontaire au combat à vitesse réelle. Le sparring est considéré comme une mise en action réelle des éléments techniques de l'expérimentation. Pour autant, ces assauts restent dans le cadre de l'exercice et visent à apporter au combattant la sensation du danger, la gestion du rythme cardiaque, etc., en le mettant en position de duelliste.

De ces assauts libres découle le principe de "tournoi», de plus en plus mis en avant. Même si le terme de compétition n'est jamais mentionné en tant que tel, chaque stage comprend un "tournoi ", qui vise à désigner le meilleur pratiquant (à l'épée longue la plupart du temps, parfois à la rapière, quelquefois avec d'autres armes) du stage, ou tout du moins, celui qui sera parvenu à vaincre le reste des participants. Les règles de ces tournois ne sont pas fixes et varient d'un stage à l'autre. Certaines associations en organisent même lors de leurs entraînements hebdomadaires. Un changement sensible se fait ressentir : au départ occasionnels, ces tournois sont devenus systématiques dans la grande majorité des stages. Aucun système de classement n'est actuellement en vigueur, mais un " prix " peut être remis au gagnant et le nom du vainqueur circule par la suite sur les forums. Bien que rejetant le principe de «sport» les pratiquants mettent en exergue le «champion ». L'image du gagnant est ainsi réutilisée sur certaines affiches de promotion de stages.

31 Les tournois sont toujours des événements centralisateurs. Ceux qui n'y prennent pas part font office de «soutien", voire de supporters. Les membres des associations sont présents pour « soutenir » leur « champion », et le tournoi prend les traits d'un véritable spectacle sportif. Le regard posé sur les gestes s'adresse particulièrement et presque uniquement à des initiés, qui connaissent et re-connaissent les coups effectués. Ce principe invite, par extension, à se questionner sur l'aspect visuel attendu des affrontements : deux enjeux sont de nouveau présents. D'une part, il existe la volonté de regarder un geste technique réussi, en ce qu'il est conforme aux textes laissés par les traités, même s'il ne suffit pas à mettre hors de combat l'adversaire. D'autre part, une recherche d'efficacité est revendiquée, même si les coups répétés sont souvent identiques, du moment qu'ils « fonctionnent ». D'où l'intérêt, lors des tournois, pour des 
touches «efficaces", qui vont augmenter les chances de "gagner ». L'orientation des techniques du côté du sport, donc de l'efficacité et de la performance ${ }^{15}$, ne risque-t-elle pas de venir transformer les pratiques premières de reconstitution et d'expérimentation? L'innovation sportive ne vient-elle pas en conflit par rapport aux enjeux liés à l'authenticité culturelle et historique des AMHE ?

Le rapport des AMHE à la démarche sportive est ambigu: d'un côté, les pratiquants rejettent cette appellation, mais de l'autre, l'intérêt accordé à la compétition, sous forme de tournoi, invite à l'inscrire dans le champ de l'espace sportif. Il existe une différence notable entre les discours et les pratiques effectives. Ceci se comprend en raison d'un changement sensible de l'activité, qui passe par une augmentation des affrontements formalisés et par une interrogation croissante de l'impact d'une éventuelle sportivisation. La question se pose de savoir si les AMHE ne sont pas, peu à peu, en train de prendre place parmi les loisirs sportifs en produisant, au-delà de la seule action physique, une pratique sportive, qui tend à répondre à la demande des adhérents. En effet, si les tournois sont en expansion, c'est bien en fonction d'une attente exprimée. L'institutionnalisation de la démarche est-elle nécessairement reliée à une forme de sportivisation? Celle-ci semble porter sur la mise en application concrète des recherches effectuées en amont, par les chercheurs ou les groupes de travail. En tant qu'objet de recherche universitaire, les AMHE ne sont pas susceptibles de devenir une pratique sportive, mais au regard de leur application technique et des innovations possibles, l'activité physique peut basculer vers l'activité sportive, du moment que des règles formelles sont instituées.

Finalement, le rapprochement entre AMHE et compétition (tournois) invite à penser que le rapport au sport est de plus en plus présent, en vue de trouver une forme de reconnaissance. Pour le dire autrement, l'attrait de la sportivisation pour les AMHE réside dans l'image sociale positive qu'elle peut donner. Les AMHE s'inscrivent donc dans un cadre moderne, qui laisse la place aux innovations sociales (loisirs) et matérielles (équipements). Le processus d'institutionnalisation et de sportivisation de la pratique est également un facteur de changement dans la conception de la démarche. De nouvelles manières de concevoir et de faire des AMHE émergent, en lien avec les transformations structurelles de la pratique (augmentation du nombre d'adhérents, évolution du marché de consommation, etc.).

\section{Conclusion}

En conclusion, la pratique actuelle des AMHE répond à plusieurs critères, allant de la reconstitution à l'innovation, en passant par l'expérimentation. Ces trois phases ( "reconstitution » de gestes techniques, « expérimentation martiale » et « innovation ») englobent des nouvelles manières de "faire une activité » historiquement située. Elles sont interdépendantes et se répondent, quelle que soit la démarche privilégiée par les associations ; elles exposent une réactualisation contemporaine d'une activité à caractère historique. La réappropriation des «arts du combat» dans le cadre du loisir contemporain présente aussi une autre manière d'aborder et de sauvegarder un patrimoine technique méconnu. 


\section{BIBLIOGRAPHIE}

BECKER Howard, «L'enquête de terrain : quelques ficelles du métier », Sociétés contemporaines, $\mathrm{n}$

- 40, octobre-décembre, 2000, p. 151-164.

COMTE Fernand et al. (sous la dir. de), L'Univers des loisirs, Paris, Letouzey et Ané, 1990.

ELIAS Norbert et DUNNING Éric, Sport et civilisation, la violence maîtrisée, trad. fr., Paris, Fayard, 1994 ( $1^{\text {re }}$ éd. allemande : 1986 ).

MUNIER Brigitte (sous la dir. de), dans MUNIER Brigitte, Sur les voies du patrimoine. Entre culture et politique, Paris, L'Harmattan, 2007.

PARLEBAS Pierre, Contribution à un lexique commenté en science de l'action motrice, Paris, INSEP, 1981.

PRUNEAU Jérôme, Les Joutes languedociennes. Ethnologie d'un « sport traditionnel », Paris,

L'Harmattan, 2003.

TEBOUL René, Culture et loisirs dans la société du temps libre, La Tour d'Aigues, Éd. de l'Aube, 2004.

TERRET Thierry, Histoire du sport, Paris, PUF, 2007.

TUAILLON DEMÉSY Audrey, La re-création du passé. Enjeux identitaires et mémoriels, Besançon, Presses universitaires de Franche-Comté, 2013.

TUAILLON DEMÉSY Audrey, «L'histoire vivante médiévale : pour une ethnographie du "passé contemporain" ", Ethnologie française, vol. 44, nº 4, octobre-décembre, 2014, p. 725-736.

\section{NOTES}

1. Pour de plus amples précisions concernant la méthodologie de recherche, nous renvoyons le lecteur à l'article suivant: TUAILLON DEMÉSY Audrey, «L'histoire vivante médiévale : pour une ethnographie du "passé contemporain" ", Ethnologie française, vol. 44, n 4, octobre-décembre, 2014, p. 725-736.

2. TUAILLON DEMÉSY Audrey, La re-création du passé. Enjeux identitaires et mémoriels, Besançon, Presses universitaires de Franche-Comté, 2013.

3. Extrait d'un entretien avec un président d'association d'AMHE (afin de respecter l'anonymat des enquêtés, les noms ne seront pas cités).

4. BECKER Howard, "L'enquête de terrain : quelques ficelles du métier ", Sociétés contemporaines, $\mathrm{n}^{\circ}$ 40, octobre-décembre, 2000, p. 162.

5. MUNIER Brigitte, «Conscience patrimoniale et sens de l'histoire », dans MUNIER Brigitte (sous la dir. de), Sur les voies du patrimoine. Entre culture et politique, Paris, L'Harmattan, 2007, p. 17.

6. UNESCO (2003), Convention pour la sauvegarde du patrimoine culturel immatériel, Paris, consultable sur http://portal.unesco.org.

7. COMTE Fernand et al. (sous la dir. de), L'Univers des loisirs, Paris, Letouzey et Ané, 1990.

8. TEBOUL René, Culture et loisirs dans la société du temps libre, La Tour d'Aigues, Éd. de l'Aube, 2004,

p. 7 .

9. http://www.amheonweb.net. 
10. ELIAS Norbert et DUNNING Éric, Sport et civilisation, la violence maîtrisée, trad. fr., Paris, Fayard, 1994 (1 $1^{\text {re }}$ éd. allemande : 1986).

11. À ce propos, voir, par exemple, PRUNEAU Jérôme, Les Joutes languedociennes. Ethnologie d'un «sport traditionnel », Paris, L'Harmattan, 2003.

12. Extrait des statuts de la FFAMHE, article 2, consultables à cette adresse: http:// www.ffamhe.fr/documents.

13. TERRET Thierry, Histoire du sport, Paris, PUF, 2007, p. 10.

14. Extrait d'un entretien avec un instructeur.

15. PARLEBAS Pierre, Contribution à un lexique commenté en science de l'action motrice, Paris, INSEP, 1981.

\section{RÉSUMÉS}

Cet article revient sur les enjeux de la pratique contemporaine des arts martiaux historiques européens (AMHE). Il aborde la question de la fidélité aux sources tout en notant l'utilité de l'expérimentation pour la reconstruction d'un geste martial authentique. Il termine sur les évolutions récentes de la pratique des AMHE, et notamment le glissement vers une pratique sportive. (résumé rédigé par la rédaction).

This paper discusses the issues of praticing historical european martial arts (HEMA) today. It begins with the issue of fidelity to the historical sources, then it notes the utility of testing, in order to rebuild an authentic martial gesture. It finish on the recent developments in the practice of HEMA, in particular the turn toward sport. (abstract written by the journal).

\section{INDEX}

Mots-clés : histoire des techniques, France, Moyen-Âge, 15e siècle, 20e siècle, 21e siècle, geste technique, expérimentation, combat

Keywords : history of technology, France, Middle Ages, 15th century, 20th century, 21st century, technical gesture, testing, fight

\section{AUTEUR}

\section{AUDREY TUAILLON DEMÉSY}

Laboratoire C3S (Culture, Sport, Santé, Société), EA 4660, Université de Franche-Comté 